\title{
Vector and mixed beams with orbital angular momentum
}

\author{
$\underline{\text { N. D. Osintseva }}^{1}$, Yu. Yu. Choporova ${ }^{1,2}$, O. E. Kameshkov ${ }^{1,2}$, B. A. Knyazev ${ }^{1,2}$, \\ V. S. Pavelyev ${ }^{3}$ \\ ${ }^{1}$ Budker Institute of Nuclear Physics SB RAS, Novosibirsk, Russia, natalyaosintseva@ gmail.com \\ ${ }^{2}$ Novosibirsk State University, Novosibirsk, Russia \\ ${ }^{3}$ Samara National Research University, Samara, Russia
}

\section{Introduction}

Vector beams with radial polarization are of great interest in surface plasmon science. Surface plasmon generation and propagation require polarization state which is perpendicular to the surface. Hence vector beams increase the generation efficiency of surface plasmon-polaritons on cylindrical metal-dielectric surfaces, such as wires [1].

In this work vector [2] and mixed beams with orbital angular momentum (OAM) [3] were generated by silicon binary diffractive optical elements (DOEs) in the $\mathrm{THz}$ range. The radiation was generated by the Novosibirsk free electron laser (NovoFEL), which has the possibility of smooth wavelength tuning from 6 to $220 \mu \mathrm{m}$ [4]. Hence, we studied the efficiency of DOEs at different wavelengths. Using Fourier filtration diffraction efficiencies of $25-30 \%$ can be achieved (except of $\lambda / 2$ and $\lambda / 4)$.

\section{Vector beams}

Three phase plates (Fig.1) transformed a linearly polarized Gaussian beam into Hermit-Gaussian (HG) modes $(1,0)$ and $(0,1)$ and into Bessel beam of first and second order. To reach a phase difference of $\pi$ at a wavelength of $141 \mu \mathrm{m}$ we used a profile height of $\Delta h=\lambda / 2(n-1)=29.1 \mu \mathrm{m}$, where $n=3.42$ is the silicon refractive index [3]. The electric field of a $\mathrm{HG}$ beam (10 or 01$)$ has opposite direction for each of its two maxima and hence, the polarization is opposite as well. Two orthogonally polarized HG beams were combined in an in-line Mach-Zehnder interferome-

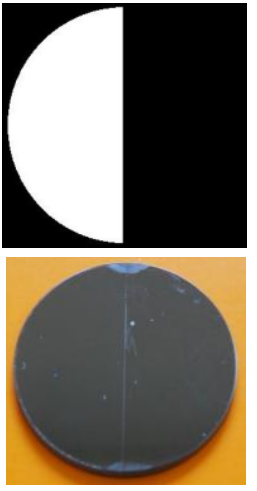

a)

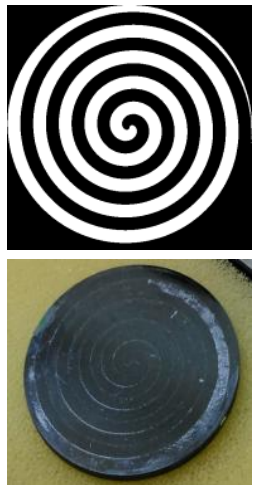

b)

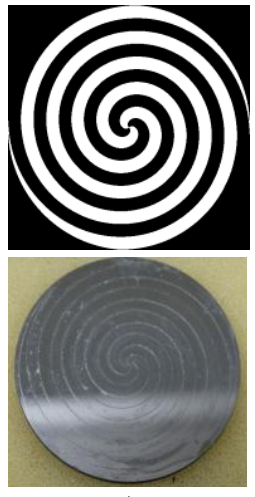

c)
Fig. 1. Diffractive optical elements for transformation $\mathrm{THz}$ Gaussian beam into Hermit-Gaussian (left column) and vortex Bessel beams with topological charges $l=1$ and $l=2$ (middle and right columns respectively). The phase distribution (top line) and photo of silicon elements (bottom line). Black zones - phase is $2 \pi N$, white zones $-\pi N$, where $N$ is integer. ter (Fig. 2). A phase singularity in the center leads to the generation of a vector Laguerre-Gaussian (LG) beam. To achieve an axially polarized beam a vertically $(1,0)$ and a horizontally polarized $(0,1) \mathrm{HG}$ beam should be combined. A radially polarized beam is formed by the superposition of orthogonal polarization of both beams (Fig. 3).

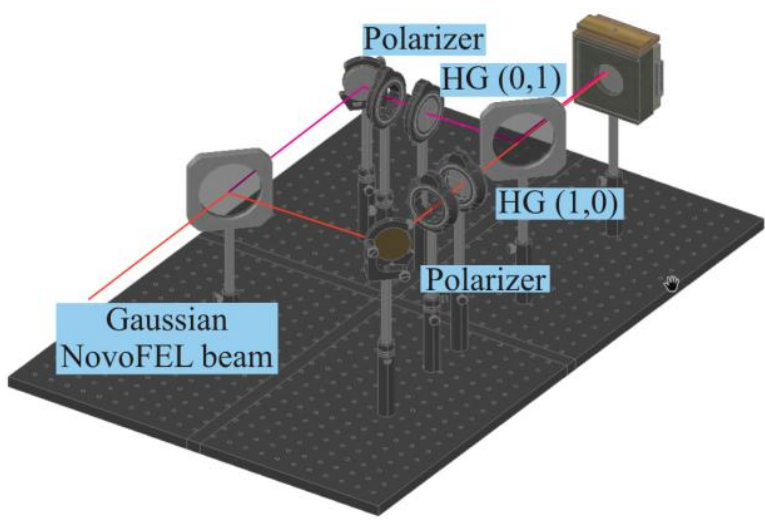

Fig. 2. The experimental scheme of generation a LaguerreGaussian vector beam. The Mach-Zehnder interferometer consists of two film beamsplitters and two mirrors. A polarizer and a DOE are situated in each arm $\mathrm{f}$ the interferometer.

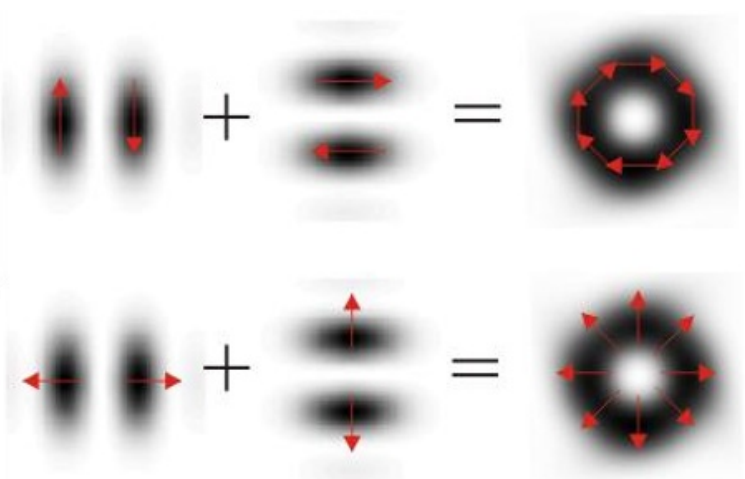

Fig. 3. Principal of generation of a vector beam. Maxima of an intensity distribution is shown with dark color. Red arrows show the direction of polarization. HG $(1,0)$ and $\operatorname{HG}(0,1)$ are used to generate Laguerre-Gaussian beam with axial (top line) and radial (bottom line) polarization.

\section{Mixed beams}

Mixed beams were generated by combination of Bessel beams with different OAM or vortex beams [6]. A helical wavefront leads to an azimuthal phase dependence in the perpendicular plane. A helical wavefront is characterized by a topological charge, or 
helicity. It is equal to the number of times the phase changes from 0 to $2 \pi$ in the perpendicular plane. Vortex Bessel beams with topological charges \pm 1 and \pm 2 were generated by phase binary axicons with spiral zones (Fig. 1) [7]. The sign of the topological charge can be changed by turning the axicon.

Interference patterns of mixed beams with different topological charges are shown in figure 4. The electric field amplitude can be written by the equation:

$$
E(r, \varphi, z)=E_{0}(r, z) e^{i\left(k_{z} z+l \varphi\right)},
$$

где $E_{0}(r, z)$ - complex amplitude, $r=\sqrt{x^{2}+y^{2}}$, $\varphi$ - azimuthal angle. Thus in a case of superposition of beams with topological charges of -1 and +1 the topological charge is equal to 0 .
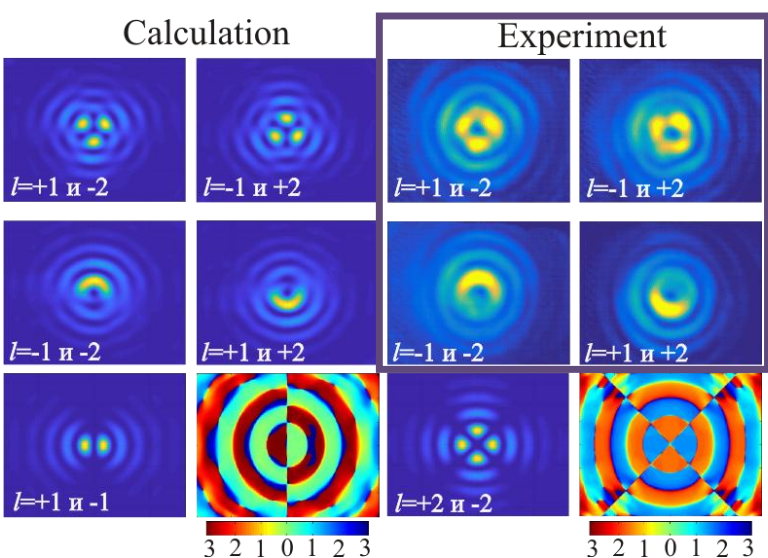

Fig. 4. Calculation and experimental results of mixed beam's generation. Bottom line: calculated intensity and phase distribution for mixed beams with equal values and different signs of topological charges.

Combinations such -1 and +2 , or +1 and -2 are more complicated and requier detailed study. If total topological charge is different from the simple \pm 1 vortex beam we can understand by using vector diffraction theory and plotting a Poynting vector (under development).

\section{References}

1. Weibin, $C$., Zhan, $Q$. Realization of an evanescent Bessel beam via surface plasmon interference excited by a radially polarized beam. // Opt. Lett. 2009. V. 34. P. 722724.

2. Oron, Ram, et al. The formation of laser beams with pure azimuthal or radial polarization. // Applied Physics Letters. 2000. V. 77. No. 21. P. 3322-3324.

3. Bouchal, Z., et al. Orbital angular momentum of mixed vortex beams. // 15th Czech-Polish-Slovak Conference on Wave and Quantum Aspects of Contemporary Optics. 2007. Vol. 6609. International Society for Optics and Photonics.

4. Volodkin, B. O., Choporova, Yu. Yu., Knyazev, B. A., et al. Fabrication and characterization of diffractive phase plates for forming high-power terahertz vortex beams using free electron laser radiation. // Opt. Quant Electron. 2016. V. 48: No. 4. P. 223.

5. Agafonov, A. N., et al. Control of transverse mode spectrum of Novosibirsk free electron laser radiation. // Applied Optics. 2015. V. 54, No. 12. P. 3635-3639.

6. Allen, L., Beijersbergen, M. W., Spreeuw, R. J. C., Woerdman, J. P. Orbital angular momentum of light and the transformation of Laguerre-Gaussian laser modes. // Phys. Rev. 1992. V. 45. P. 8185-8189.

7. Choporova, $Y u$. Yu., et al. High-power terahertz nondiffractive bessel beams with angular orbital momentum: Generation and application. // IRMMW-THz 2015: 40th International Conference on Infrared, Millimeter, and Terahertz waves. Hong Kong, 23 - 28 August 2015. - Piscataway: IEEE, 2015. - Art.nr 7327684. DOI 10.1109/IRMMW-THz.2015.7327684. 